\title{
Avaliação de repertórios de contar e recontar histórias em crianças
}

Daniela Mendonça Ribeiro1. Universidade Federal de Alagoas.

Ana Carolina Sella. Universidade Federal de Alagoas.

Carmen Sílvia Motta Bandini. Centro Universitário CESMAC; Universidade Estadual de Ciências da Saúde de Alagoas.

Heloísa Helena Motta Bandini. Universidade Estadual de Ciências da Saúde de Alagoas.

\section{Resumo}

Os estudantes brasileiros apresentam um dos piores desempenhos em tarefas de compreensão de textos em avaliações como o Programa Internacional de Avaliação de Alunos (PISA), o que sugere que procedimentos específicos devem ser desenvolvidos para remediar seus déficits. O desenvolvimento desses procedimentos, por sua vez, requer uma avaliação sistemática de repertórios relacionados ao contar e recontar histórias, para que se identifiquem objetivos de ensino específicos e individualizados. Este estudo avaliou as habilidades de contar e recontar histórias de crianças, bem como alguns de seus pré-requisitos, por meio de um conjunto de testes padronizados e de tarefas elaboradas para a avaliação destas habilidades. Os desempenhos foram analisados de acordo com características macro e microestruturais da linguagem. Os dados mostram que as tarefas foram eficazes na caracterização dos repertórios de contação e recontagem de histórias, fornecendo dados suficientes para a formulação de programas de ensino individualizado.

Palavras-chave: avaliação; produção; histórias; crianças.

\begin{abstract}
Assessing story production and retelling repertoires in children. Brazilian students had one of the worst performances in reading comprehension tasks in assessments such as the Programme for International Student Assessment (PISA), which suggests that specific procedures should be designed to remediate their deficits. This, in turn, requires a systematic evaluation of repertoires related to storytelling and retelling, so that specific and individualized teaching goals can be targeted. This study assessed children storytelling and retelling skills, as well as some of their prerequisites, through a set of standardized tests and tasks developed to evaluate these skills. Performance was analyzed according to linguistic macro and microstructures. Data show that the tasks were efficient in characterizing storytelling and retelling repertoires, providing enough data to design individualized teaching programs.
\end{abstract}

Keywords: assessment; production; stories; children.

\section{Resumen}

La evaluación de los repertorios de contar y de volver a contar historias en niños. Estudiantes brasileños tienen un de los peores rendimientos en tareas de comprensión de lectura en evaluaciones como el Programa Internacional de Evaluación de los Alumnos (PISA), lo que sugiere que se deben desarrollar procedimientos específicos para la remediación de esos déficits. El desarrollo de esos procedimientos, a su vez, requiere una evaluación sistemática de repertorios relacionados con contar y volver a contar historias, con el fin de identificar objetivos educativos específicos e individualizados. Eso estudio evaluó la capacidad de contar y volver a contar historias de los niños, así como algunos de sus requisitos previos, a través de una serie de testes estandarizados y tareas diseñadas para evaluar esas habilidades. Los rendimientos fueron analizados de acuerdo a las características macro y microestructurales de idioma. Los datos muestran que las tareas propuestas fueron eficaces en la caracterización de los repertorios de contar y volver a contar historias, proporcionando datos suficientes para formular programas de aprendizaje individualizados. Palabras clave: evaluación; producción; historias; niños.

\footnotetext{
${ }^{1}$ Na época em que o estudo foi conduzido, a autora era docente do Centro Universitário CESMAC de Maceió.
} 
A aquisição de habilidades ${ }^{1}$ linguísticas, como produzir e compreender histórias, tem consequências importantes em quase todos os aspectos da vida de uma criança, incluindo interações positivas com seus pares, comunicação eficiente e aprendizagem adequada na escola (Spencer \& Slocum, 2010). Por outro lado, crianças com habilidades linguísticas pouco desenvolvidas podem apresentar problemas comportamentais e sociais (Botting \& Conti-Ramsden, 2000), assim como dificuldades na leitura (Snow, Burns, \& Griffin, 1998).

A despeito da importância dos repertórios de produzir e de compreender histórias orais e escritas, dados recentes sobre o desempenho de estudantes brasileiros indicam que eles estão aquém do esperado para suas idades e níveis de escolaridade. De acordo com o Programa Internacional de Avaliação de Alunos (PISA) de 2012, o desempenho em compreensão de leitura de nossos estudantes do $7^{\circ}$ ano em diante, com idade aproximada de 15 anos, revela que eles são capazes apenas de identificar o tema principal ou o objetivo do autor em um texto sobre um tópico familiar e de realizar conexões simples entre a informação apresentada no texto e o conhecimento do dia-a-dia (OECD, 2012). Estes dados evidenciam a necessidade de se desenvolver procedimentos específicos para a remediação dos déficits de compreensão de textos dos estudantes brasileiros. Tal desenvolvimento, por sua vez, requer uma avaliação sistemática dos repertórios de produção e de compreensão de textos orais e escritos, incluindo comportamentos de contar e recontar histórias, a fim de se identificar objetivos de ensino específicos. Isso porque não é possível criar objetivos acadêmicos sem a descrição em termos concretos e mensuráveis daquilo que compõe o repertório alvo final (Vargas, 2009).

$\mathrm{Na}$ literatura sobre avaliação de habilidades de contação e de recontagem de histórias, encontram-se estudos conduzidos a partir de dois principais propósitos. Um destes é o de medir habilidades linguísticas de crianças com desenvolvimento típico e com deficiências, assim como identificar os déficits característicos dos diferentes tipos de atraso de linguagem (p. ex., Befi-Lopes, Bento, \& Perissinoto, 2008; Booting, 2002). O outro propósito é o de analisar e comparar os desempenhos em tarefas de contação e de recontagem por crianças de diferentes idades, níveis de escolaridade e classes sociais (p. ex., Brandão \& Spinillo, 2001; Pessoa, Correa, \& Spinillo, 2010).
Para exemplificar o primeiro conjunto de estudos, Booting (2002) utilizou narrativas para diferenciar padrões similares e diferentes de prejuízos, decorrentes da forma mais comum do distúrbio específico de linguagem (DEL) e do distúrbio semântico-pragmático severo. Os participantes foram expostos a duas condições. $\mathrm{Na}$ primeira, foi apresentado um livro de história ilustrado que não continha palavras escritas e o pesquisador, após contar a história mostrando as figuras aos participantes, solicitava a sua recontagem, usando as figuras como dicas. A segunda condição foi de produção de história a partir de outro livro ilustrado. Foram analisadas variáveis tais como o número de palavras e de frases completas, os erros de conjugação verbal, a utilização de dispositivos narrativos (termos mentalísticos, afetivos e sociocognitivos), bem como a estrutura das histórias (abertura formal, orientação do leitor para os personagens e ambiente, menção explícita ao tema, resolução e finalização formal). Os resultados mostraram que, em ambos os grupos, as histórias produzidas foram maiores, em termos de números de palavras, do que as recontadas. Em relação à estrutura das histórias, os participantes com distúrbio semântico-pragmático inseriram mais elementos do que os participantes com DEL. O autor concluiu que, para crianças com DEL, a habilidade linguística pode ser o principal obstáculo e, no caso das crianças com distúrbio semântico-pragmático, estas possuem as habilidades sociocognitivas e pragmáticas afetadas, o que compromete a utilização dos dispositivos narrativos.

Para ilustrar o conjunto de estudos interessados na comparação dos desempenhos de crianças de diferentes idades, níveis de escolaridade e classes sociais, Brandão e Spinillo (2001) verificaram as relações entre a produção e a compreensão de textos em: (1) diferentes faixas etárias; (2) relação à estrutura linguística textual; e (3) reprodução, considerando a relação com o texto original no que se refere à fidelidade das ideias apresentadas no texto. Participaram do estudo 60 crianças, que foram igualmente distribuídas em três grupos de acordo com a idade: 4, 6 e 8 anos. Cada participante foi exposto a duas sessões: na primeira sessão, foi aplicada uma tarefa de contação e, na segunda, uma tarefa de recontagem. Na tarefa de contação, os participantes foram solicitados a contar uma história a partir de um tema e, na tarefa de recontagem, eles foram instruídos a recontar uma história apresentada pela pesquisadora. Os resultados mostraram que as produções dos participantes de 8 anos foram mais elaboradas do que as dos de 4 
anos. Os participantes de 6 anos mostraram um padrão de desempenho mais próximo ao do grupo de 4 anos. Resultados semelhantes foram observados na tarefa de recontagem, exceto pelo fato de que o desempenho dos participantes de 6 anos foi mais próximo ao desempenho dos participantes de 8 anos. Não foi detectada correlação entre as habilidades de produção e de compreensão. As autoras concluíram que as relações entre produção e compreensão são diferentes ao longo do desenvolvimento: entre crianças mais jovens, é mais fácil produzir um texto original do que reproduzir um texto ouvido e, à medida que as crianças se tornam mais velhas, esta relação se inverte, tornando-se mais fácil demonstrar compreensão do que produzir um novo texto.

Os estudos descritos acima apresentaram tarefas para a avaliação dos comportamentos de contar e recontar histórias de crianças com desenvolvimento típico (Brandão \& Spinillo, 2001) e com atraso de linguagem (Booting, 2002). Em ambos os estudos, foram apresentadas dicas para a realização das tarefas: Booting apresentou um livro ilustrado e Brandão e Spinillo forneceram um tema a partir do qual os participantes contaram uma história. Outra semelhança entre os estudos refere-se ao fato de que foi fornecida apenas uma oportunidade para os participantes realizarem cada tarefa. As avaliações apresentadas por esses estudos podem gerar duas críticas. A primeira refere-se à restrição do tema na tarefa de contação de histórias. Embora Brandão e Spinillo argumentem que o tema foi fornecido para delimitar o conhecimento dos participantes, pode-se especular que a restrição da produção a um dado tema pode mascarar sua habilidade de produzir histórias criativas e originais. Além disso, conforme relatado na literatura, a utilização de figuras como dicas nas tarefas de contação pode levar a produções mais elementares (Spinillo \& Pinto, 1994). Outra crítica refere-se ao número de oportunidades que cada participante teve em cada condição dos estudos: fornecer mais de uma oportunidade para a emissão dos comportamentos permitiria que se verificasse com mais precisão seus repertórios. Isso porque o participante pode não compreender exatamente o que se espera dele na primeira vez em que é exposto a uma dada tarefa, dentre outras variáveis que podem interferir no desempenho em um dia específico.

A partir dessas considerações, o objetivo deste estudo foi avaliar as habilidades de contar e recontar histórias de crianças, bem como alguns de seus pré-requisitos, por meio de um conjunto de testes padronizados e de tarefas elaboradas para a avaliação destas habilidades. Os testes padronizados foram aplicados para avaliar a memória em diferentes níveis e a inteligência não verbal, em especial a atenção, pré-requisitos das habilidades em questão (p. ex., Gathercole, 1999). Histórias foram elaboradas de modo a apresentar suas categorias estruturais de maneira balanceada (Petersen, Gillam, Spencer, \& Gillam, 2010) e os desempenhos nas tarefas de contação e de recontagem foram medidos mais de uma vez.

\section{Método}

\section{Participantes}

Participaram do estudo sete crianças, cinco meninas e dois meninos, com idades entre 10 e 11 anos, estudantes do $6^{\circ}$ ano do Ensino Fundamental de uma escola particular da região Nordeste do Brasil. As sete crianças foram indicadas por seus professores e atenderam ao critério para participação no estudo: desempenho médio ou acima da média no Teste de Matrizes Progressivas de Raven. Os testes de Audibilização (Golbert, 1988) e de Aprendizagem Auditivo-Verbal (Rey, 1964) foram aplicados para caracterizar os participantes (Tabela 1).

\section{Ambiente e Materiais}

O estudo foi conduzido individualmente em uma sala da escola. Foram utilizados: (a) um iPad (Marca Apple, modelo A1395) para a apresentação das histórias nas tarefas de recontagem e de jogos eletrônicos; (b) uma câmera fotográfica (Marca Sony, modelo shot dsc w530); e (c) um gravador digital (Marca Sony, modelo ICD-PX312F) para registro das histórias contadas e recontadas. Nas tarefas de recontagem, foram utilizadas duas histórias, planejadas de modo a incluir os conteúdos relativos a todas as categorias estruturais de histórias na sequência esperada pela comunidade verbal (Personagem, Local e Tempo - compõem o Cenário -, Tema, Enredo e Resolução), segundo a classificação de Ribeiro, Pascualon, Sella, Bandini e de Souza (2009). Em ambas as histórias, cada categoria foi apresentada o mesmo número de vezes, sendo que as categorias Personagem, Local e Tempo foram apresentadas duas vezes cada e as categorias Tema, Enredo e Resolução foram apresentadas uma vez cada, para evitar que a diferença no número de apresentações das categorias em cada história interferisse no desempenho (p. ex., Ribeiro et al., 2009). 
Avaliação de repertórios de contar e recontar histórias em crianças

Tabela 1. Caracterização dos Participantes.

\begin{tabular}{|c|c|c|c|c|c|}
\hline Participante & Gênero & Idade $^{a}$ & Raven & $\begin{array}{l}\text { Teste de Aprendizagem } \\
\text { Auditivo-Verbal }\end{array}$ & Teste de audibilização \\
\hline 1 & Feminino & 10,9 & Definidamente acima da média na capacidade intelectual & Ausência de perda & Grupo superior \\
\hline 2 & Masculino & 11,3 & Intelectualmente médio & Ausência de perda & Grupo superior \\
\hline 3 & Feminino & 11,3 & Intelectualmente superior & Ausência de perda & Grupo médio superior \\
\hline 4 & Masculino & 11,4 & Intelectualmente médio & Perda severa & Grupo médio superior \\
\hline 5 & Feminino & 11,4 & Intelectualmente médio & Ausência de perda & Grupo superior \\
\hline 6 & Feminino & 11,6 & Definidamente acima da média na capacidade intelectual & Ausência de perda & Grupo médio superior \\
\hline 7 & Feminino & 11,8 & Intelectualmente superior & Ausência de perda & Grupo superior \\
\hline
\end{tabular}

${ }^{a}$ Em anos e meses no início do estudo.

\section{Procedimento}

Inicialmente, as crianças foram convidadas a participar do estudo e brincaram com jogos eletrônicos para estabelecimento de rapport. Em seguida, os testes e as tarefas de contação e de recontagem foram aplicados na seguinte ordem: (a) teste de Matrizes Progressivas de Raven; (b) duas tarefas de contação de histórias livre; (c) teste de Aprendizagem Auditivo-Verbal; (d) teste de Audibilização; e (e) duas tarefas de recontagem de histórias. Os testes foram aplicados individualmente e cada teste foi aplicado em dias diferentes, a fim de que as tarefas não se tornassem cansativas para os participantes e que a exposição a um dos testes não interferisse no desempenho em outro teste. Ao final de cada dia de coleta de dados, os participantes tiveram acesso a um jogo eletrônico de sua preferência, independentemente de seu desempenho na tarefa realizada. Seguem as descrições de cada etapa do procedimento.

Teste de Matrizes Progressivas de Raven. Avalia a atenção concentrada de crianças de 5 a 11 anos de idade. Este teste foi aplicado porque estudos como o de Stipek e Valentino (2015) mostram que a atenção é um importante preditor do desempenho em tarefas de compreensão de texto em crianças pequenas. Para garantir tal pré-requisito, apenas crianças com desempenho médio ou acima da média foram incluídas no estudo.

Teste de Aprendizagem Auditivo-Verbal. Este teste foi aplicado por medir a memória recente e de reconhecimento e a susceptibilidade à interferência para retenção após outras atividades, habilidades envolvidas em tarefas de recontagem (p. ex., Uehara \& Landeira-Fernandez, 2010). A classificação do desempenho seguiu os critérios descritos em Andersson et al. (2006).

Teste de Audibilização. Verifica o nível de habilidades de discriminação fonética, memória auditivo-verbal e conceituação. A análise dos resultados baseou-se nos níveis de classificação estabelecidos para crianças com
7 anos de idade, visto que não há normatização para indivíduos acima dessa idade (Silva \& Golbert, 2011). Este teste foi aplicado por avaliar habilidades correlacionadas a desempenhos em contação e em recontagem de histórias, conforme descrito anteriormente.

Tarefas de Contação de Histórias Livre. Em cada uma dessas tarefas, os participantes foram solicitados a contar uma história, após a instrução: "Eu gosto muito de ouvir histórias. Você poderia me contar uma história?". Se o participante não iniciasse uma história após 10 segundos, a instrução "Você poderia me contar uma história?" era repetida. Nenhuma outra dica foi apresentada.

Tarefas de Recontagem de Histórias. Inicialmente, era apresentada a instrução: "Eu vou te contar uma história. Por favor, escute com atenção. Quando eu terminar, você me contará a mesma história. Podemos começar?". Após a apresentação da história por meio do iPad, a pesquisadora dizia: "Agora você pode me contar a história".

\section{Procedimento de Análise dos Resultados}

As histórias contadas e recontadas foram transcritas e analisadas de acordo com suas macro e microestruturas linguísticas, conforme definidas por Petersen et al. (2010).

A macroestrutura das histórias foi analisada de acordo com: (1) inserção das categorias e sequência em que foram apresentadas e (2) construção do episódio. Além disso, identificaram-se os assuntos principais das histórias contadas pelos participantes. A análise da inserção das categorias permitiu a identificação da organização da história em termos de elementos indicativos de proficiência narrativa (Petersen et al., 2010). Nas tarefas de contação e de recontagem, analisou-se a inserção das categorias Personagem, Local, Tempo, Tema, Enredo e Resolução (Ribeiro et al., 2009) e a sequência em que elas foram apresentadas. Nas tarefas de recontagem, analisou-se também a acurácia com que cada uma das 
categorias apresentadas nas histórias ouvidas foi inserida, sendo que 1 ponto foi atribuído à inserção completa, 0,5 ponto foi atribuído à incompleta e 0 pontos foi atribuído à não inserção da categoria. A análise da construção do episódio baseou-se nas categorias propostas por Applebee (1978) e interpretadas por Petersen et al. (2010). Cada uma das histórias foi classificada em sequência descritiva, sequência de ações, sequência reativa, episódio incompleto, episódio completo ou múltiplos episódios².

As microestruturas foram analisadas de acordo com: (1) número total de palavras; (2) porcentagem de palavras diferentes; e (3) inserção de conjunções coordenativas: aditivas (p. ex., e, nem, não só... mas também), adversativas (p. ex., mas, porém), alternativas (p. ex., ou... ou, ora... ora), conclusivas (p. ex., logo, por isso) e explicativas (p. ex., que, porque) e de conjunções subordinativas: temporais (p. ex., quando, antes, depois), causais (p. ex., porque, visto que), comparativas (p. ex., que, do que), concessivas (p. ex., embora, ainda que), conformativas ( $p$. ex., como, segundo), consecutivas ( $p$. ex., tanto, tão), finais (p. ex., para que) e proporcionais (p. ex., à medida que, quanto mais). A inserção das conjunções foi analisada porque elas revelam dados acerca da complexidade e coesão de histórias.

\section{Concordância entre Observadores}

Dentre as 28 histórias contadas e recontadas, $36 \%$ (10 histórias) foram enviadas a um observador independente que analisou as variáveis referentes às macro e microestruturas de cada história. O índice de concordância foi calculado de acordo com cada variável dependente. Em relação à inserção das categorias nas tarefas de contação, à sequência em que elas foram apresentadas nas duas tarefas e à análise da construção do episódio, fez-se a divisão do número de concordâncias pela soma do número de concordâncias mais o número de discordâncias multiplicado por 100. No que se refere à inserção das categorias nas tarefas de recontagem, ao número total e de palavras diferentes e à inserção das conjunções, dividiu-se o menor número de registros pelo maior número de registros multiplicado por 100 . O índice de concordância foi de $88,86 \%$ (variação entre $83,3 \%$ e $100 \%$ ) para a inserção das categorias nas tarefas de contação e de $85,62 \%$ ( $75 \%$ a $100 \%$ ) nas tarefas de recontagem, $100 \%$ para a sequência em que elas foram apresentadas nas duas tarefas, $90 \%$ para a construção do episódio, $100 \%$ para o número total de palavras, $95,43 \%$ $(88,60 \%$ e $100 \%)$ para o número de palavras diferentes e $91,40 \%$ (84,61\% e 100\%) para a inserção das conjunções.

\section{Resultados}

\section{Tarefas de Contação de Histórias Livre}

Em relação ao assunto geral das histórias, a maioria delas (oito) tratava de questões fantasiosas, tipicamente presentes em contos de fadas, como uma princesa mimada, que se perdeu em uma floresta e encontrou um duende que a aconselhou a deixar de ser mimada (primeira história contada por P1). Outras quatro histórias tratavam de questões que poderiam estar presentes no cotidiano dos participantes, como uma menina que sente ciúmes de sua irmã mais velha e esconde seu celular (segunda história contada por P1). E duas histórias (contadas por P4) apresentavam características antropomórficas, tais como uma vaca desastrada e seu amigo sapo que foram a uma festa de aniversário.

No que se refere à presença e à sequência de inserção das categorias (Tabela 2), nove histórias apresentaram cinco categorias (primeira história de P1, P2, P3, P4, P5 e P6 e segunda história de P2, P4 e P5), quatro apresentaram quatro categorias (primeira história de $\mathrm{P} 7$ e segunda história de P1, P3 e P7) e apenas uma apresentou todas as categorias (segunda história de P6). Contudo, a inserção das categorias não implicou maior complexidade das histórias: a segunda história de P6, única que continha as seis categorias, foi classificada como sequência de ações na análise de construção de episódio, enquanto três das quatro histórias classificadas como episódios completos, continham apenas quatro categorias (segunda história de $\mathrm{P} 1, \mathrm{P} 3$ e de P7). Em relação à sequência de apresentação das categorias, em todas as histórias, elas foram inseridas na sequência esperada: Personagem, Local e Tempo (Cenário) poderiam ser apresentadas em qualquer ordem desde que aparecessem no início da história e antes das categorias Tema, Enredo e Resolução, sendo que estas últimas deveriam aparecer nesta sequência.

Em relação às microestruturas, na primeira história contada por cada participante, o número de palavras variou entre 166 (P4) e 499 (P6) (Tabela 3). Na segunda, todos os participantes contaram histórias mais longas (variação entre 180 (P4) e 1556 (P6) palavras). No que se refere à porcentagem de palavras diferentes em relação ao número total de palavras, na primeira história, esta variou entre $25,45 \%$ (P6) e $40,69 \%$ (P2) e, na segunda, entre $15,93 \%$ (P6) e $39,44 \%$ (P4). Ao se relacionar estas variáveis, verifica-se que $\mathrm{P} 4$, embora tenha contado as histórias mais curtas, foi quem utilizou um maior número de palavras diferentes na segunda história, enquanto P6, 
Avaliação de repertórios de contar e recontar histórias em crianças

Tabela 2. Presença e Sequência das Categorias Estruturais e Nível de Classificação na Análise da Construção do Episódio, nas Tarefas de Contação e de Recontagem de Histórias.

\begin{tabular}{|c|c|c|c|c|}
\hline \multirow{3}{*}{ Participantes } & \multicolumn{4}{|c|}{ Presença e sequência das categorias estruturais e nível de construção do episódio } \\
\hline & \multicolumn{4}{|c|}{ Tarefas de contação } \\
\hline & Primeira história & Segunda história & & \\
\hline 1 & $\mathrm{P} ; \mathrm{L} ; \mathrm{T} ; \mathrm{E} ; \mathrm{R}$ & sequência de ações & $\mathrm{P} ; \mathrm{T} ; \mathrm{E} ; \mathrm{R}$ & episódio completo \\
\hline 2 & $\mathrm{P} ; \mathrm{L} ; \mathrm{T} ; \mathrm{E} ; \mathrm{R}$ & episódio completo & $\mathrm{P} ; \mathrm{L} ; \mathrm{T} ; \mathrm{E} ; \mathrm{R}$ & episódio completo \\
\hline 3 & $P ; T ; L ; E ; R$ & sequência de ações & $\mathrm{P} ; \mathrm{T} ; \mathrm{E} ; \mathrm{R}$ & episódio completo \\
\hline 4 & $P ; L ; T ; E ; R$ & sequência de ações & $\mathrm{P} ; \mathrm{L} ; \mathrm{T} ; \mathrm{E} ; \mathrm{R}$ & sequência de ações \\
\hline 5 & P; Tp; T; E; R & sequência de ações & $\mathrm{P} ; \mathrm{L} ; \mathrm{T} ; \mathrm{E} ; \mathrm{R}$ & episódio completo \\
\hline 6 & $\mathrm{P} ; \mathrm{T} ; \mathrm{L} ; \mathrm{E} ; \mathrm{R}$ & sequência de ações & Tp; P; L; T; E; R & sequência de ações \\
\hline 7 & $\mathrm{P} ; \mathrm{T} ; \mathrm{E} ; \mathrm{R}$ & sequência de ações & P; T; E; R & episódio completo \\
\hline \multicolumn{5}{|c|}{ Tarefas de recontagem } \\
\hline 1 & P; Tp; T; E; R & sequência de ações & $\mathrm{P} ; \mathrm{T} ; \mathrm{E} ; \mathrm{R}$ & sequência de ações \\
\hline 2 & $P ; L ; E ; R$ & sequência de ações & $\mathrm{P} ; \mathrm{T} ; \mathrm{E} ; \mathrm{R}$ & sequência de ações \\
\hline 3 & P; Tp; E; R & sequência de ações & L; P; Tp; T; E; R & sequência de ações \\
\hline 4 & P; Tp; E; R & sequência de ações & P; L; T; E; R & sequência de ações \\
\hline 5 & P; Tp; E; R & episódio completo & $\mathrm{P} ; \mathrm{T} ; \mathrm{E} ; \mathrm{R} ; \mathrm{Tp}$ & sequência descritiva \\
\hline 6 & Tp; P; E; R & sequência de ações & Tp; P; L; T; E; R & sequência de ações \\
\hline 7 & L; P; T; E; R & sequência de ações & Tp; P; L; T; E; R & sequência de ações \\
\hline
\end{tabular}

P: personagem, L: local, Tp: tempo; T: tema; E: enredo; R: resolução.

Tabela 3. Número Total e Número de Palavras Diferentes Apresentadas em Cada Uma das Histórias Contadas e Recontadas por Cada Participante.

\begin{tabular}{|c|c|c|c|c|c|}
\hline \multirow{2}{*}{ Participante } & \multirow{2}{*}{ Tarefa } & Total de palavras & Palavras diferentes & Total de palavras & Palavras diferentes \\
\hline & & \multicolumn{2}{|c|}{ Tarefas de Contação } & \multicolumn{2}{|c|}{ Tarefas de Recontagem } \\
\hline \multirow{2}{*}{1} & 1. ${ }^{\mathrm{a}}$ & 182 & $70(38,46 \%)$ & 207 & $79(38,16 \%)$ \\
\hline & $2 .^{a}$ & 207 & $81(39,13 \%)$ & 180 & $71(39,44 \%)$ \\
\hline \multirow{2}{*}{2} & $1 .^{a}$ & 172 & $70(40,69 \%)$ & 131 & $62(47,32 \%)$ \\
\hline & $2 .^{a}$ & 295 & $99(33,55 \%)$ & 137 & $60(43,79 \%)$ \\
\hline \multirow{2}{*}{3} & 1. ${ }^{\mathrm{a}}$ & 203 & $82(40,39 \%)$ & 138 & $64(46,37 \%)$ \\
\hline & 2. ${ }^{a}$ & 232 & $87(37,50 \%)$ & 177 & $62(35,02 \%)$ \\
\hline \multirow{2}{*}{4} & $1 .^{a}$ & 166 & $56(33,73 \%)$ & 156 & $62(39,74 \%)$ \\
\hline & $2 .^{\text {a }}$ & 180 & $71(39,44 \%)$ & 105 & $52(49,52 \%)$ \\
\hline \multirow{2}{*}{5} & $1 .{ }^{a}$ & 307 & $120(39,08 \%)$ & 215 & $90(41,86 \%)$ \\
\hline & 2. ${ }^{a}$ & 946 & $224(23,67 \%)$ & 164 & $79(48,17 \%)$ \\
\hline \multirow{2}{*}{6} & 1. ${ }^{\mathrm{a}}$ & 499 & $127(25,45 \%)$ & 254 & $92(36,22 \%)$ \\
\hline & 2. ${ }^{a}$ & 1556 & $248(15,93 \%)$ & 205 & $81(39,51 \%)$ \\
\hline \multirow{2}{*}{7} & 1. ${ }^{\mathrm{a}}$ & 367 & $99(26,97 \%)$ & 186 & $83(44,62 \%)$ \\
\hline & 2. ${ }^{\mathrm{a}}$ & 474 & $152(32,06 \%)$ & 172 & $70(40,69 \%)$ \\
\hline
\end{tabular}

As porcentagens entre parênteses indicam a porcentagem de palavras diferentes em relação ao número total de palavras de cada história.

que contou as histórias mais longas, foi quem inseriu o menor número de palavras diferentes nas duas histórias.

Os resultados relativos à inserção das conjunções são apresentados na Tabela 4. As conjunções que não foram inseridas em nenhuma história foram omitidas desta tabela. As conjunções mais utilizadas foram: a explicativa que (199 vezes) e a aditiva e (191 vezes), inseridas nas duas histórias por todos os participantes. A conjunção adversativa mas foi a terceira mais utilizada (45 vezes) e inserida pelos participantes em, pelo menos, uma história. As conjunções menos utilizadas foram: a conclusiva por isso e a temporal antes (duas vezes cada), a causal uma vez que, a comparativa que e a consecutiva que (uma vez cada). 


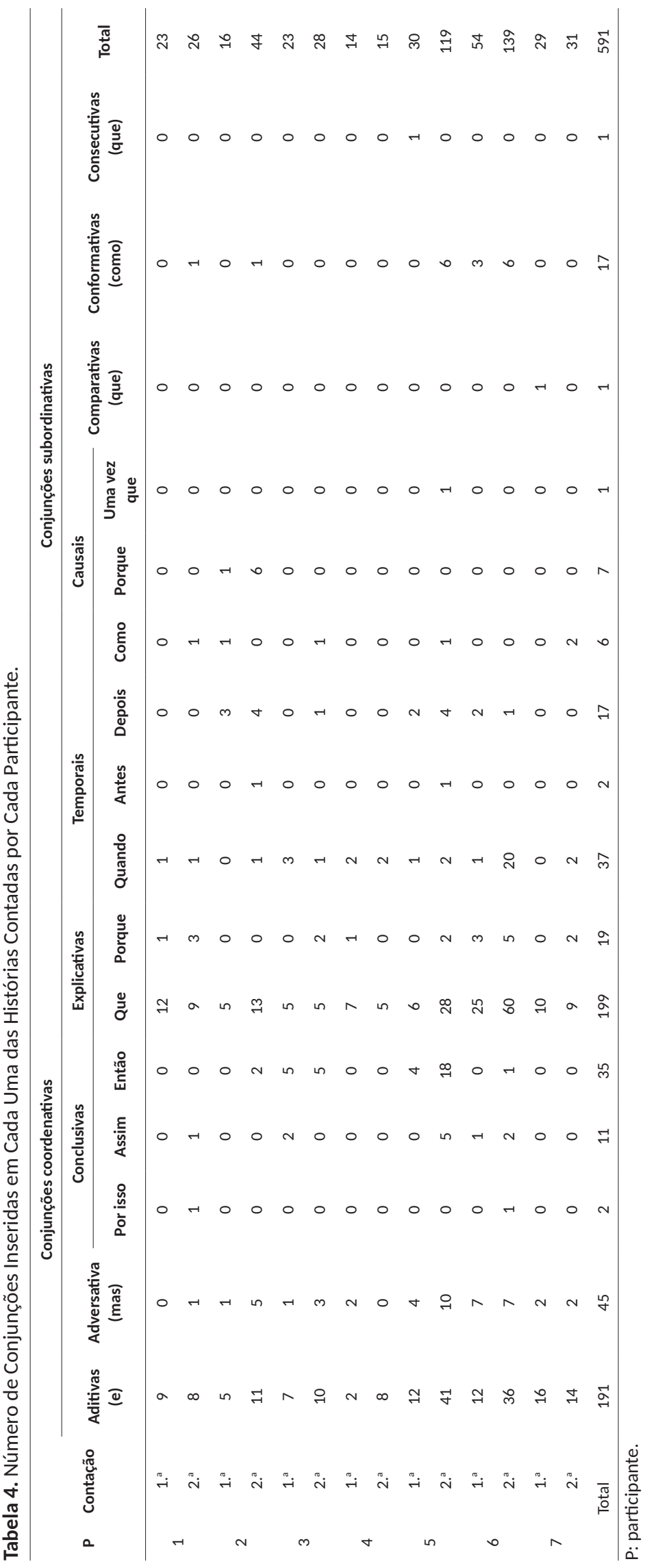




\section{Tarefas de Recontagem de Histórias}

Em relação à presença e à sequência de apresentação das categorias (Tabela 2), sete histórias apresentaram quatro categorias (primeira história de P2, P3, P4, P5 e P6 e segunda história de P1 e P2), quatro apresentaram cinco categorias (primeira história de P1 e P7 e segunda história de P4 e P5) e três apresentaram todas as categorias (segunda história de P3, P6 e P7). Quando estes dados são comparados aos das tarefas de contação, verifica-se que o número histórias que apresentaram seis categorias foi maior nesta tarefa (três em recontagem e uma em contação), contudo foi maior também o número de histórias que apresentaram quatro categorias (sete em recontagem e quatro em contação). No que se refere à sequência de apresentação das categorias, em quase todas as histórias, elas foram inseridas na sequência ideal, exceto na segunda história de P5, em que a categoria Tempo foi inserida ao final.

Em relação à complexidade das histórias, assim como nas tarefas de contação, a inserção das categorias não implicou histórias mais complexas: as três histórias que apresentaram as seis categorias foram classificadas como sequência de ações e o único episódio completo (primeira história de P5) apresentou quatro categorias.

No que se refere à acurácia com que as categorias foram inseridas (Tabela 5), observa-se que, quando as categorias foram inseridas, elas estavam, em sua maioria, completas (1 ponto). Em outras palavras, considerando todas as histórias recontadas, 66 categorias foram inseridas, sendo que 50 ( $75,8 \%$ ) delas estavam completas, enquanto $16(24,2 \%)$ estavam incompletas. Além disso, quando esta variável foi considerada nas duas histórias recontadas por cada participante, não houve diferença entre o número de categorias completas e incompletas apresentado na primeira e na segunda histórias. Por exemplo, considerando as duas histórias recontadas, P1 sempre inseriu categorias completas, enquanto P3, P4 e P6 inseriram categorias completas e incompletas. Por outro lado, P2, P5 e P7 inseriram categorias completas e incompletas na primeira história e apenas completas na segunda.

Em relação às microestruturas, vale ressaltar que a primeira história contada aos participantes continha 285 palavras e a segunda, 313. Na primeira história recontada, o número de palavras variou entre 131 (P2) e 254 (P6) e, na segunda, entre 105 (P4) e 205 (P6). No que se refere ao uso de palavras diferentes, a porcentagem variou entre $36,22 \%$ (P6) e $47,32 \%$ (P2) na primeira história e entre $35,02 \%$ (P3) e 49,52\% (P4) na segunda (Tabela 3). Esses resultados foram semelhantes aos das
Tabela 5. Acurácia com que Cada Categoria Estrutural Foi Inserida em Cada História nas Tarefas de Recontagem de Histórias.

\begin{tabular}{|c|c|c|c|c|c|c|c|}
\hline \multirow{2}{*}{ Participante } & \multirow{2}{*}{ História } & \multicolumn{6}{|c|}{ Categorias } \\
\hline & & $\mathbf{P}$ & $\mathbf{L}$ & $\mathrm{Tp}$ & $\mathbf{T}$ & $\mathrm{E}$ & $\mathbf{R}$ \\
\hline \multirow{2}{*}{1} & $1^{\mathrm{a}}$ & 1 & 0 & 1 & 1 & 1 & 1 \\
\hline & $2^{\mathrm{a}}$ & 1 & 0 & 0 & 1 & 1 & 1 \\
\hline \multirow{2}{*}{2} & $1^{\mathrm{a}}$ & 1 & 1 & 0 & 0 & 0,5 & 1 \\
\hline & $2^{\mathrm{a}}$ & 1 & 0 & 0 & 1 & 1 & 1 \\
\hline \multirow{2}{*}{3} & $1^{a}$ & 1 & 0 & 0,5 & 0 & 0,5 & 1 \\
\hline & $2^{\mathrm{a}}$ & 0,5 & 1 & 1 & 1 & 0,5 & 1 \\
\hline \multirow{2}{*}{4} & $1^{\mathrm{a}}$ & 0,5 & 0 & 1 & 0 & 0,5 & 1 \\
\hline & $2^{\mathrm{a}}$ & 1 & 1 & 0 & 0,5 & 1 & 0,5 \\
\hline \multirow{2}{*}{5} & $1^{a}$ & 1 & 0 & 0,5 & 0 & 0,5 & 1 \\
\hline & $2^{\mathrm{a}}$ & 1 & 0 & 1 & 1 & 1 & 1 \\
\hline \multirow{2}{*}{6} & $1^{\mathrm{a}}$ & 1 & 0 & 0,5 & 0 & 1 & 1 \\
\hline & $2^{\mathrm{a}}$ & 1 & 1 & 1 & 0,5 & 0,5 & 0,5 \\
\hline \multirow{2}{*}{7} & $1^{\mathrm{a}}$ & 0,5 & 1 & 0 & 1 & 1 & 1 \\
\hline & $2^{\mathrm{a}}$ & 1 & 1 & 1 & 1 & 1 & 1 \\
\hline
\end{tabular}

0: categoria não inserida na história; 0,5: categoria inserida de maneira incompleta na história; 1: categoria inserida de maneira completa na história.

tarefas de contação: os participantes P4 e P2 produziram histórias mais curtas em ambas as tarefas e com a maior porcentagem de palavras diferentes. Por outro lado, P6 produziu as histórias mais longas em ambas as tarefas e com o menor número de palavras diferentes na segunda tarefa de recontagem. De maneira geral, as histórias foram mais curtas e com maior número de palavras diferentes nas tarefas de recontagem quando comparadas as de contação.

Em relação à análise da inserção das conjunções (Tabela 6), assim como nas tarefas de contação, a conjunção aditiva e e a explicativa que foram as mais utilizadas (102 e 93 vezes, respectivamente), sendo inseridas nas duas histórias por todos os participantes. As conjunções menos utilizadas foram a alternativa ou...ou, a conclusiva assim, a temporal antes ( 2 vezes cada), as causais como e já que e a comparativa que (1 vez cada).

\section{Discussão e conclusões}

O objetivo do presente estudo foi avaliar as habilidades de contar e recontar histórias de crianças, bem como alguns de seus pré-requisitos, por meio de um conjunto de testes padronizados e de tarefas elaboradas para a avaliação destas habilidades. De maneira geral, as tarefas mostraram-se úteis para tal finalidade, revelando algumas dificuldades dos participantes em relação à organização de suas ideias durante as tarefas. 
D.M. Ribeiro, A.C. Sella, C.S.M. Bandini, H.H.M. Bandini

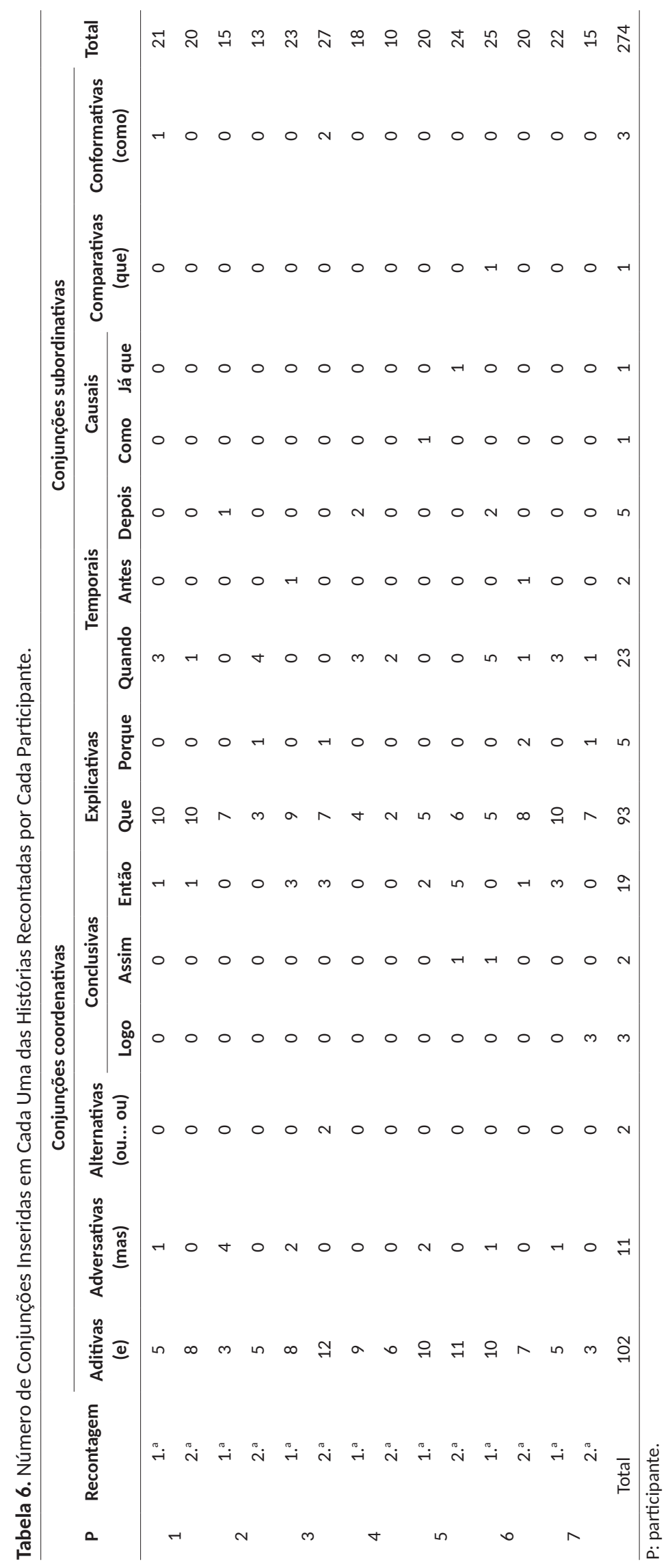


Uma dessas dificuldades foi demonstrada pela relação entre a inserção das categorias estruturais e a complexidade da construção do episódio. Considerando todas as histórias contadas e recontadas, foram inseridas, pelo menos, quatro categorias em cada história, sendo que três delas (Personagem, Enredo e Resolução) estavam presentes em todas as histórias. Apenas quatro histórias continham as seis categorias, sendo uma história produzida nas tarefas de contação e três, nas de recontagem. Embora essas histórias estivessem completas em termos da inserção das categorias, elas foram classificadas como sequência de ações em relação à construção de episódio. Isso porque elas continham conjunções que relacionavam temporalmente, mas não causalmente as ações do(s) personagem(s). Por outro lado, algumas das histórias que continham quatro categorias foram classificadas como episódios completos, pois relacionavam temporal e causalmente as ações dos personagens, sendo que essas ações eram direcionadas para a resolução de um problema e coerentes com este problema. Apesar destas análises (inserção das categorias e construção de episódio), referirem-se à complexidade das histórias, elas se baseiam em parâmetros que revelam diferentes níveis de habilidades (Hayward \& Schneider, 2000). O principal desses parâmetros refere-se à utilização das conjunções, principalmente, temporais e causais, que são fundamentais para a proficiência narrativa (Petersen et al., 2010). Na análise de construção de episódio, a inserção dessas conjunções foi um dos critérios para que as histórias fossem classificadas em níveis mais complexos. Por outro lado, a ausência de conjunções temporais e causais caracterizou histórias mais elementares, que foram classificadas como sequências descritivas e de ações, respectivamente, mesmo que elas apresentassem todas as categorias estruturais.

Os resultados revelam o nível dos repertórios dos participantes no momento de aplicação das tarefas e corroboram evidências de que as habilidades narrativas continuam sendo aprimoradas ao longo da vida (p. ex., Principe \& Smith, 2008). Outra possibilidade de interpretação dos resultados baseia-se na literatura que afirma que o desenvolvimento das habilidades narrativas alcança o mais alto nível de complexidade em torno dos 10 anos de idade (p. ex., Izard et al., 2001). É importante ressaltar que a interpretação dos resultados obtidos caberá ao pesquisador ou ao professor que aplicar as tarefas, visto que ele analisará os dados a partir das concepções teóricas que guiam o seu trabalho.
Uma análise detalhada dos dados relativos à inserção das conjunções revela um predomínio na utilização de conjunções coordenativas em detrimento das conjunções subordinativas. Tal predomínio pode ser compreendido considerando-se que as conjunções coordenativas expressam relações mais simples entre eventos e orações, enquanto que as subordinativas ligam elementos de níveis sintáticos diferentes, em que uma das orações é membro sintático da outra (Bechara, 2010). Durante a aquisição da linguagem, observa-se, inicialmente, a utilização de conjunções aditivas e, posteriormente, de conjunções que expressam relações adversativas, temporais e causais. Aos 3 anos, espera-se que a criança utilize essas conjunções de forma flexível (Araujo, 2007). No entanto, os resultados deste estudo corroboram outros que sugerem que o uso das conjunções ainda está em evolução por volta dos 10 anos de idade (p. ex., Bento \& Befi-Lopes, 2010).

No que se refere à relação entre medidas de produtividade da linguagem (número total e porcentagem de palavras diferentes) e complexidade das histórias, há evidências, na literatura, de que, à medida que as crianças se tornam mais velhas, suas histórias tornam-se mais complexas, mais longas e apresentam mais palavras diferentes (Justice et al., 2006). Os resultados deste estudo demonstram que o número de palavras diferentes parece estar mais relacionado à complexidade das histórias do que ao número total de palavras. Por exemplo, dentre as sete histórias classificadas como episódios completos, quatro delas apresentavam porcentagem de palavras diferentes próxima à porcentagem máxima identificada nas histórias produzidas em cada tarefa (primeira história contada por P2, segunda, por P1 e P3, e primeira história recontada por P5). Estes resultados corroboram sugestões de que o número de palavras diferentes é uma medida que reflete não só a produtividade da linguagem, mas também habilidades semânticas (Mäkinen, Loukusa, Nieminen, Leinonen, \& Kunnari, 2014), visto que mais palavras diferentes implica a apresentação de informações mais significativas.

Uma análise da relação entre os desempenhos nas tarefas de contação e de recontagem revela que, de forma geral, os participantes inseriram mais categorias, produziram histórias mais complexas, com maior número de palavras e maior variedade de conjunções no primeiro tipo de tarefa. Esses resultados contrariam a hipótese de que, entre crianças mais jovens, é mais fácil produzir um texto original do que reproduzir um texto 
ouvido, sendo que, com o avanço da idade, esta relação se inverte, sendo mais fácil demonstrar compreensão do que produzir um novo texto (Brandão \& Spinillo, 2001). As habilidades envolvidas nas tarefas de contação e de recontagem estão relacionadas à produção de texto, no entanto, na recontagem, o novo texto deve estar relacionado ao texto original. Portanto, a recontagem depende também de habilidades de memória, pois é necessário que, ao ouvir a história, as informações principais sejam retidas e, posteriormente, reproduzidas de modo a manter a sua fidedignidade (Oakhill, 1984). A exigência de se manter fiel às informações da história ouvida pode ter restringido a recontagem dos participantes, levando-os a recontarem histórias mais curtas e menos complexas em termos de construção de episódio e da inserção das categorias.

O papel da memória na recontagem também pode ser observado ao se verificar os resultados do Teste de Aprendizagem Auditivo-Verbal. Embora testes estatísticos não tenham sido conduzidos, P2 e P5 produziram as histórias mais complexas de acordo com a análise de construção do episódio e obtiveram a segunda e a terceira pontuações mais altas, respectivamente, neste teste. De maneira análoga, P4 foi classificado com perda severa de memória no teste, o que pode ter afetado seu desempenho na recontagem. Uma ampla discussão sobre o papel da memória em tarefas de linguagem pode ser encontrada em Gathercole (1999) e em estudos subsequentes (p. ex., Uehara \& Landeira-Fernandez, 2010).

A partir dos aspectos mencionados acima, verifica-se que as tarefas propostas para avaliar o contar e o recontar histórias são viáveis para tal finalidade, uma vez que fornecem uma ampla variedade de informações relevantes sobre os repertórios dos participantes. Em termos da elaboração das tarefas, este estudo avança em relação a estudos anteriores conduzidos para avaliar a contação e a recontagem em, pelo menos, dois aspectos. O primeiro refere-se ao fato de a tarefa de contação de histórias ter sido livre, o que pode ter ampliado as possibilidades de os participantes apresentarem seu melhor desempenho, e o segundo refere-se ao fornecimento de duas oportunidades para emissão de cada comportamento, diminuindo a possibilidade de incompreensão da tarefa e os efeitos de possíveis variáveis intervenientes.

Estas tarefas podem ser aplicadas por professores e pesquisadores interessados no delineamento de intervenções para remediar déficits nos repertórios de contar e recontar histórias. É importante ressaltar que, neste estudo, a análise dos resultados considerou todas as variáveis identificadas na literatura como importantes para a proficiência narrativa. Entretanto, a escolha das variáveis a serem analisadas dependerá do interesse de quem aplicar as tarefas, bem como a decisão sobre aplicar os testes de memória e de inteligência não verbal. Neste estudo, os testes foram aplicados para caracterizar os participantes em relação a essas habilidades, pois elas são consideradas pré-requisitos para o contar e recontar histórias.

A aplicação destas tarefas por professores e pesquisadores fornecerá informações para a definição de objetivos e planejamento de ensino específicos às necessidades dos estudantes. É importante que estudos futuros relacionem os dados obtidos com a aplicação das tarefas propostas pelo presente estudo com dados relacionados à idade, condições socioeconômicas, vivências na família e na escola a fim de se obter uma avaliação ecológica, visto que estas variáveis também exercem influência sobre as habilidades de contar e recontar histórias.

\section{Referências}

Andersson, C., Lindau, M., Almkvist, O., Engfeldt, P., Johansson, S. E., \& Jönhagen, M. E. (2006). Identifying patients at high and low risk of cognitive decline using Rey Auditory Verbal Learning Test among middle-aged memory clinic outpatients. Dementia and Geriatric Cognitive Disorders, 21(4), 251-259. doi: 10.1159/000091398

Applebee, A. N. (1978). A child's concept of story. Chicago: University of Chicago Press.

Araujo, K. (2007). Desempenho gramatical de criança em desenvolvimento normal e com distúrbio específico de linguagem. (Tese de doutorado). Recuperado de http://www.teses.usp.br/teses/disponiveis/8/8139/tde-10102007-151501/pt-br.php

Bechara, E. (2010). Gramática escolar da língua portuguesa. (2a ed.). Rio de Janeiro: Nova Fronteira.

Befi-Lopes, D. M., Bento, A. C. P., \& Perissinoto, J. (2008). Narração de histórias por crianças com distúrbio específico de linguagem. Pró-Fono Revista de Atualização Científica, 20(2), 93-98. doi: 10.1590/S0104-56872008000200004

Bento, A. C., \& Befi-Lopes, D. M. (2010). Story organization and narrative by school-age children with typical language development. Pró-Fono Revista de Atualização Científica, 22(4), 503-508. doi: 10.1590/ S0104-56872010000400024

Botting, N. (2002). Narrative as a tool for the assessment of linguistic and pragmatic impairments. Child Language Teaching and Therapy, 18(1), 17-31. doi: 10.1191/0265659002ct224oa

Botting, N., \& Conti-Ramsden, G. (2000). Social and behavioral difficulties in children with language impairment. Child Language Teaching and Therapy, 16(2), 105-120. doi: 10.1177/026565900001600201

Brandão, A. C. P., \& Spinillo, A. G. (2001). Produção e compreensão de textos em uma perspectiva de desenvolvimento. Estudos de Psicologia, 6(1), 51-62. doi: 10.1590/S1413-294X2001000100006 
Gathercole, S. (1999). Cognitive approaches to the development of short-term memory. Trends in Cognitive Sciences, 3(11), 410-419. doi: 10.1016/S1364-6613(99)01388-1

Golbert, C. S. (1988). A evolução psicolinguística e suas implicações na alfabetização: teoria, avaliação, reflexões. Porto Alegre: Artes Médicas.

Hayward, D. V., \& Schneider, P. (2000). Effectiveness of teaching story grammar knowledge to pre-school children with language impairment: An exploratory study. Child Language Teaching and Therapy, 16(3), 255-284. doi: 10.1177/026565900001600303

Izard, C.E., Fine S., Schultz, D., Mostow, A., Ackerman, B., \& Youngstrom, E. (2001). Emotional knowledge as a predictor of social behavior and academic competence in children at risk. Psychological Science, 12(1), 18-23. doi: 10.1111/1467-9280.00304

Justice, L. M., Bowles, R. P., Kaderavek, J. N., Ukrainetz, T. A., Eisenberg, S. L., \& Gillam, R. B. (2006). The index of narrative microstructure: a clinical tool for analyzing school-aged children's narrative performances. American Journal of Speech-Language Pathology, 15, 177-191. doi: 10.1044/1058-0360(2006/017)

Mäkinen, L., Loukusa, S., Nieminen, L., Leinonen, E., \& Kunnari, S. (2014). The development of narrative productivity, syntactic complexity, referential cohesion and event content in four- to eight-year-old Finnish children. First Language, 34, 24-42. doi: $10.1177 / 0142723713511000$

Oakhill, J. (1984). Inferencial and memory skills in children's comprehension of stories. British Journal of Educational Psychology, 54, 31-39. doi: 10.1111/j.2044-8279.1984.tb00842.x

Pessoa, A. P., Correa, J., \& Spinillo, A. G. (2010). Contexto de produção e o estabelecimento da coerência na escrita de histórias por crianças. Psicologia: Reflexão e Crítica, 23(2), 253-60. doi: 10.1590/S010279722010000200007

Petersen, D. B., Gillam, S. L., Spencer, T. D., \& Gillam, R. B. (2010). The effects of literate narrative intervention on children with neurologically based language impairments: an early stage study. Journal of Speech, Language, and Hearing Research, 53, 961-981. doi: 10.1044/1092-4388(2009/09-0001)

Principe, G. F., \& Smith, E. (2008). The tooth, the whole tooth, and nothing but the tooth: How belief in the tooth fairy can engender false memories. Applied Cognitive Psychology, 22(5), 625-642. doi: 10.1002/acp.1402
Rey, A. (1964). L'Examen clinique em psychologie. Paris: Press Universitaire de France.

Ribeiro, D. M., Pascualon, J. F., Sella, A.C., Bandini, C.S. M., \& de Souza, D. G. (2009). Avaliação de um procedimento para ensinar categorias estruturais de histórias. Revista Psicologia Escolar e Educacional, 13(2), 303-314. doi: 10.1590/s1413-85572009000200013

Santos, G. C.V., Kienen, N., Viecili, J., Botomé, S.P., \& Kubo, O. M. (2009). "Habilidades" e "competências" a desenvolver na capacitação de psicólogos: uma contribuição da Análise do Comportamento para o exame das Diretrizes Curriculares. Interação em Psicologia, 13(1), 131-145. doi: 10.5380/psi.v13il.12279

Silva, S. F., \& Golbert, C.S. (2011, novembro). Avaliação das dificuldades de aprendizagem na matemática em alunos da $2^{a}$ série ( $3^{\circ}$ ano) do Ensino Fundamental. Comunicação apresentada no X Congresso Nacional de Educação - EDUCERE, Curitiba.

Snow, C. E., Burns, M. S., \& Griffin, P. (Orgs.). (1998). Preventing reading difficulties in young children. Washington: National Academy Press.

Spencer, T. D., \& Slocum, T. A. (2010). The effect of a narrative intervention on story retelling and personal story generation skills of preschoolers with risk factors and narrative language delays. Journal of Early Intervention, 32(3), 178-199. doi: 10.1177/1053815110379124

Spinillo, A. G., \& Pinto, G. (1994). Children's narratives under different conditions: a comparative study. British Journal of Developmental Psychology, 12(2), 177-193. doi: 10.1111/j.2044-835X.1994. tb00627.x

Stipek, D., \& Valentino, R. A. (2015). Early childhood memory and attention as predictors of academic growth trajectories. Journal of Educational Psychology, 107(3), 771-788. doi: 10.1037/edu0000004

The Organization for Economic Co-operation and Development (2012) Education at a Glance: OECD Indicators 2012 - Brazil. Retrieved from http://www.oecd.org/brazil/EAG2012\%20-\%20Country\%20 note\%20-\%20Brazil.pdf

Uehara, E., \& Landeira-Fernandez, J. (2010). Um panorama sobre o desenvolvimento da memória de trabalho e seus prejuízos no aprendizado escolar. Ciências e Cognição, 15(2), 31-41. Recuperado de http://www.cienciasecognicao.org/revista/index.php/cec/article/ view/375/187

Vargas, J. S. (2009). Behavior analysis for effective teaching. Nova lorque: Routeledge.

\footnotetext{
${ }^{1}$ Compreende-se por habilidades "comportamentos ou conjuntos de comportamentos que caracterizam determinado desempenho do indivíduo" (Santos, Kienen, Viecili, Botomé, \& Kubo, 2009, p. 133-134).

${ }^{2}$ A classificação das histórias em cada um desses níveis foi baseada em suas definições operacionais, que foram descritas em termos mensuráveis para aumentar a probabilidade de objetividade da análise e da concordância entre observadores.
} 
D.M. Ribeiro, A.C. Sella, C.S.M. Bandini, H.H.M. Bandini

Daniela Mendonça Ribeiro, Doutora em Educação Especial pela Universidade Federal de São Carlos (UFSCar), Pós-doutorado em Psicologia pela UFSCar, é Professora Adjunto A, na Universidade Federal de Alagoas (UFAL). Endereço para correspondência: Av.

Dr. Antônio Gouveia, 475, ap. 105, Pajuçara. CEP: 57030-170. Maceió - AL. E-mail: danimribeiro@yahoo.com.br

Ana Carolina Sella, Doutora em Educação Especial pela Universidade Federal de São Carlos (UFSCar), Pós-doutorado em Psicologia Comportamental para Transtornos do Espectro do Autismo, pelo Centro Médico da Universidade do Nebraska (UNMC), Munroe-Meyer Institute, Center for Autism Spectrum

Disorders, é Professora Adjunto A, na Universidade Federal de Alagoas (UFAL). E-mail: carolsella@yahoo.com.br

Carmen Silvia Motta Bandini, Doutora em Filosofia pela Universidade Federal de São Carlos (UFSCar), é Professora Titular

III no Centro Universitário CESMAC (CESMAC) e Professora

Adjunta na Universidade Estadual de Ciências da Saúde de Alagoas (UNCISAL). E-mail: csmbandini@gmail.com

Heloísa Helena Motta Bandini, Doutora em Educação Especial, pela Universidade Federal de São Carlos (UFSCar), Pós-doutorado em Educação Especial, pela UFSCAR, é Professora

Titular da Disciplina de Aparelhos de Amplificação Sonora Individual da Universidade Estadual de Ciências da Saúde de Alagoas (UNCISAL). E-mail: heloisabandini@gmail.com 